\title{
Ccceative
commons
}

Also available at http://amc-journal.eu

ISSN 1855-3966 (printed edn.), ISSN 1855-3974 (electronic edn.)

ARS MATHEMATICA CONTEMPORANEA 13 (2017) 15-21

\section{The distinguishing index of the Cartesian product of countable graphs}

\author{
Izak Broere * \\ Department of Mathematics and Applied Mathematics, University of Pretoria \\ Monika Pilśniak ${ }^{\dagger}$ \\ AGH University, Department of Discrete Mathematics, Krakow, Poland
}

Received 6 January 2015, accepted 12 July 2016, published online 11 August 2016, corrected 12 January 2017

\begin{abstract}
The distinguishing index $D^{\prime}(G)$ of a graph $G$ is the least cardinal $d$ such that $G$ has an edge colouring with $d$ colours that is preserved only by the trivial automorphism.

We derive some bounds for this parameter for infinite graphs. In particular, we investigate the distinguishing index of the Cartesian product of countable graphs.

Finally, we prove that $D^{\prime}\left(K_{2}^{\aleph_{0}}\right)=2$, where $K_{2}^{\aleph_{0}}$ is the infinite dimensional hypercube.

Keywords: Distinguishing index, automorphism, infinite graph, edge colouring, infinite dimensional hypercube.

Math. Subj. Class.: 05C25, 05C80, 03E10

\section{Introduction}

Albertson and Collins [1] introduced the (vertex-)distinguishing number $D(G)$ of a graph $G$ as the least cardinal $d$ such that $G$ has a labelling with $d$ labels that is only preserved by the trivial automorphism. This concept has spawned numerous papers, mostly on finite graphs. But countable infinite graphs have also been investigated with respect to the distinguishing number; see [12], [13], and [14]. For graphs of higher cardinality, see [8]. The corresponding notion for endomorphisms instead of automorphisms is investigated in [5].

Let us consider now any edge colouring of a graph $G$; it is merely a function $f$ : $E(G) \rightarrow C$ which labels each edge of $G$ with a colour from some set $C$. Given a graph

\footnotetext{
* This author is thankful to the AGH University of Science and Technology whose hospitality he enjoyed during the preparation of this paper; he is also supported in part by the National Research Foundation of South Africa (Grant Numbers 90841, 91128).

${ }^{\dagger}$ The research was partially supported by the Polish Ministry of Science and Higher Education.

E-mail addresses: izak.broere@up.ac.za (Izak Broere), pilsniak@agh.edu.pl (Monika Pilśniak)
} 
$G$ with an edge colouring $f$, we say that a graph automorphism $\varphi: V(G) \rightarrow V(G)$ of $G$ preserves the edge colouring $f$ if $f(x y)=f(\varphi(x) \varphi(y))$ for every edge $x y \in E(G)$; if, on the other hand, there is an edge $x y$ such that $f(x y) \neq f(\varphi(x) \varphi(y))$, then we say that $\varphi$ is broken by $x y$. It is easy to see that there is, for every connected graph $G \neq K_{2}$, an edge colouring of $G$ which is preserved only by the trivial automorphism of $G$, i.e., only by the identity $\operatorname{id}_{G}: V(G) \rightarrow V(G)$ : Merely choose different colours for different edges. The distinguishing index $D^{\prime}(G)$ of a graph $G$ is the least cardinal $d$ such that $G$ has an edge colouring with $d$ colours that is only preserved by the trivial automorphism. Obviously for $K_{2}$ the distinguishing index is not defined and it is the only such connected graph.

For finite graphs this concept is investigated by Kalinowski and Pilśniak in [9] and by Pilśniak in [11]. In [2], the following general upper bound was proved.

Theorem 1.1. Let $G$ be a connected, infinite graph such that the degree of every vertex of $G$ is not greater than $\Delta$. Then $D^{\prime}(G) \leq \Delta$.

A graph $G$ is said to be prime with respect to the Cartesian product if whenever $G \cong$ $G_{1} \square G_{2}$, then either $G_{1}$ or $G_{2}$ is the graph consisting of a single vertex. It is well known (see [6]) that if $G$ is connected, then $G$ has a unique prime factorization, i.e.,

$$
G \cong G_{1} \square G_{2} \square \cdots \square G_{t}
$$

such that for $1 \leq i \leq t, G_{i}$ is prime. Two graphs $G$ and $H$ are called relatively prime if $K_{1}$ is the only common factor of $G$ and $H$. About forty-five years ago Imrich and Miller independently proved the following theorem - see Thm. 6.10 in [6].

Theorem 1.2. If $G$ is connected and $G=G_{1} \square G_{2} \square \cdots \square G_{r}$ is its prime decomposition, then every automorphism of $G$ is generated by the automorphisms of the factors and the transpositions of isomorphic factors.

A basic fact, which is a reformulation of the above theorem for $r=2$ and which is used frequently in this paper, is:

If $\varphi$ is an automorphism of the Cartesian product $G_{1} \square G_{2}$ of two connected relatively prime graphs, then there are automorphisms $\varphi_{i}$ of $G_{i}, i=1,2$, such that $\varphi\left(v_{1}, v_{2}\right)=$ $\left(\varphi_{1}\left(v_{1}\right), \varphi_{2}\left(v_{2}\right)\right)$ for all $\left(v_{1}, v_{2}\right) \in V\left(G_{1} \square G_{2}\right)$.

In this case we write $\varphi=\left(\varphi_{1}, \varphi_{2}\right)$ for short and we note that $\varphi$ is non-trivial if and only if at least one of $\varphi_{1}$ and $\varphi_{2}$ is non-trivial.

An asymmetric graph has only one automorphism, the trivial automorphism. We now state an easy corollary of these properties and definitions for product graphs with distinguishing index 1.

Proposition 1.3. Let $G$ be the Cartesian product of two graphs $G_{1}$ and $G_{2}$. Then

$$
D^{\prime}\left(G_{1} \square G_{2}\right)=1
$$

if and only if $G_{1}$ and $G_{2}$ are relatively prime and both are asymmetric graphs.

The aim of this paper is to present new results for the distinguishing index of the Cartesian product of infinite graphs. Most graphs in this document are countable, i.e., finite or denumerable; numbers used are either finite or $\aleph_{0}$.

Subgraphs of the Cartesian product $G_{1} \square G_{2}$ of the form $G_{1} \square\{v\}$ (for any $v \in V\left(G_{2}\right)$ ) are isomorphic to $G_{1}$ and are called $G_{1}$-layers of $G_{1} \square G_{2}$. The $G_{2}$-layers of $G_{1} \square G_{2}$ are defined similarly. 
The distinguishing index of the Cartesian product of finite graphs is investigated in [4] where the authors prove, amongst others, a result which will be useful in the next section and which we now record as

Theorem 1.4. Let $G$ be a connected finite graph and $k \geq 2$. Then $D^{\prime}\left(G^{k}\right)=2$ with the only exception: $D^{\prime}\left(K_{2}^{2}\right)=3$.

\section{The distinguishing index of the Cartesian product}

First we consider the Cartesian product of two denumerable graphs with infinite edge sets.

Lemma 2.1. Let $G_{1}$ and $G_{2}$ be two connected relatively prime denumerable graphs. Then $D^{\prime}\left(G_{1} \square G_{2}\right) \leq 2$.

Proof. We start by labelling the edges of $G_{1}$ with $e_{1}, e_{2}, \ldots$ and those of $G_{2}$ with $f_{1}, f_{2}, \ldots$ This is possible since both edge sets have to be denumerable. Note that these labellings effectively order the edges of these graphs. We can now easily describe the required edge distinguishing colouring in colours 1 and 2:

Colour the first (in terms of the above ordering) $k$ edges of the $k$ 'th layer of $G_{1}$ and the first $k$ edges of the $k$ 'th layer of $G_{2}$ with 1 ; colour all other edges with 2 . Recall that every edge in $G_{1} \square G_{2}$ lies in a $G_{1}$-layer or a $G_{2}$-layer; hence this process colours indeed all edges of $G_{1} \square G_{2}$. Using the labels, this means that the edges corresponding to the edges $\left\{e_{1}, e_{2}, \ldots, e_{k}\right\}$ of $G_{1}$ in the $k$ 'th $G_{1}$-layer and the edges corresponding to the edges $\left\{f_{1}, f_{2}, \ldots, f_{k}\right\}$ of $G_{2}$ in the $k$ 'th $G_{2}$-layer, for all $k=1,2, \ldots$, are coloured 1 and all other edges are coloured 2.

Now consider, if possible, any non-trivial automorphism $\varphi=\left(\varphi_{1}, \varphi_{2}\right)$ of $G_{1} \square G_{2}$ which preserves the above edge colouring of $G_{1} \square G_{2}$. Since every two different $G_{1}$-layers have different numbers of edges coloured with 1 , the automorphism $\varphi_{2}$ of $G_{2}$ must be trivial. Similarly, $\varphi_{1}$ must be trivial. Hence $\varphi$ is the trivial automorphism, proving that for every non-trivial automorphism $\varphi$ of $G_{1} \square G_{2}$ there is an edge $e$ of $G_{1} \square G_{2}$ for which $e$ and $\varphi(e)$ are coloured differently.

The same result was obtained for the distinguishing number of two connected relatively prime denumerable graphs by Imrich and Klavžar in [7]. Recently it was shown by Estaji, Imrich, Kalinowski, Pilśniak and Tucker in [3] that the condition that the two graphs are relatively prime can be omitted.

Note that Lemma 2.1 assures us that $D^{\prime}\left(G_{1} \square G_{2}\right)$ is at most two irrespective of the values of $D^{\prime}\left(G_{1}\right)$ and $D^{\prime}\left(G_{2}\right)$. Next we consider the case in which both $G_{1}$ and $G_{2}$ of orders being any cardinals and with finite values for the distinguishing index.

Lemma 2.2. Suppose $G_{1}$ and $G_{2}$ are connected relatively prime graphs with finite distinguishing indexes. If $D^{\prime}\left(G_{i}\right) \leq k_{i}, i=1,2$, then $D^{\prime}\left(G_{1} \square G_{2}\right) \leq \max \left\{k_{1}, k_{2}\right\}$.

Proof. Since $D^{\prime}\left(G_{i}\right) \leq k_{i}, i=1,2$, there are, with $k=\max \left\{k_{1}, k_{2}\right\}$, edge colourings $f_{1}$ of $G_{1}$ and $f_{2}$ of $G_{2}$ using the colours $1,2, \ldots, k$ which are distinguishing colourings of $G_{1}$ and $G_{2}$ respectively. In order to prove now that $D^{\prime}\left(G_{1} \square G_{2}\right) \leq k$, we again use the notion of a "first" layer through a labelling of the vertices (which here is not explicitly chosen or named). Hence consider the function $f: E\left(G_{1} \square G_{2}\right) \rightarrow\{1,2, \ldots, k\}$ defined by

1) $f\left(\left(v_{1}, w\right)\left(v_{2}, w\right)\right)=f_{1}\left(v_{1} v_{2}\right)$ for edges of the first $G_{1}$-layer and 
2) $f\left(\left(v, w_{1}\right)\left(v, w_{2}\right)\right)=f_{2}\left(w_{1} w_{2}\right)$ for edges of the first $G_{2}$-layer and

3) $f(e)=1$ for all remaining edges.

Consider any non-trivial automorphism $\alpha=\left(\alpha_{1}, \alpha_{2}\right)$ of $G_{1} \square G_{2}$ with $\alpha_{1}$ a non-trivial automorphism of $G_{1}$ or $\alpha_{2}$ a non-trivial automorphism of $G_{2}$. Assume that the first is true (for $G_{1}$ ): Then, since $f_{1}$ is a distinguishing colouring of the first $G_{1}$-layer, there is an edge $e$ of $G_{1}$ such that $f_{1}(e) \neq f_{1}\left(\alpha_{1}(e)\right)$. Now, if $\alpha_{2}$ does not move the first layer, then this edge (considered as an edge of $G_{1} \square G_{2}$ ) is an edge of the required kind in the first $G_{1}$-layer. On the other hand, if $\alpha_{2}$ does move the first layer to another layer, we can remark, since $f_{1}(e) \neq f_{1}\left(\alpha_{1}(e)\right)$, that at least one of $f_{1}(e)$ and $f_{1}\left(\alpha_{1}(e)\right)$ is different from 1 so that this edge is moved by $\alpha_{2}$ to an edge in another layer which has colour 1 by 3) above.

A similarly argument holds if the second is true (for $G_{2}$ ) - merely interchange the roles of $G_{1}$ and $G_{2}$ (and their colourings and automorphisms) in the above argument.

Hence we are assured that all non-trivial automorphisms of $G_{1} \square G_{2}$ are broken by the colouring $f$.

Observe, that $D^{\prime}\left(G_{1} \square G_{2}\right)$ can be arbitrary large, for instance if $G_{1}$ is isomorphic to $P_{3}$ and $G_{2}$ is isomorphic to an infinite ray with many (but finitely many) leaves adjacent to its first vertex.

In our next result we prove that if $G_{1}$ satisfies $D^{\prime}\left(G_{1}\right)=\aleph_{0}$ and the graph $G_{2}$ is finite (so that, in particular $D^{\prime}\left(G_{2}\right)$ is finite), then $D^{\prime}\left(G_{1} \square G_{2}\right)=\aleph_{0}$.

Lemma 2.3. Suppose $G_{1}$ and $G_{2}$ are connected relatively prime graphs with $D^{\prime}\left(G_{1}\right)=\aleph_{0}$ and $G_{2}$ is finite. Then $D^{\prime}\left(G_{1} \square G_{2}\right)=\aleph_{0}$.

Proof. Suppose, for a proof by contradiction, that $D^{\prime}\left(G_{1} \square G_{2}\right)$ is finite. Since $G_{2}$ is a finite graph, there are finite values for $\left\|G_{2}\right\|$, the number of edges of $G_{2}$, and $D^{\prime}\left(G_{2}\right)$ too. Hence we can choose a positive integer $k$ such that each of these three numbers is at most $k$.

Since $D^{\prime}\left(G_{1} \square G_{2}\right) \leq k$, there is a $k$-distinguishing edge colouring $f$ of the edges of $G_{1} \square G_{2}$. Furthermore, since $D^{\prime}\left(G_{1}\right)=\aleph_{0}$, there exists, for every positive integer $t$, a nontrivial automorphism $\alpha_{t}$ of $G_{1}$ which needs at least $t+1$ colours to break it. So if $t \geq k$, the colouring by $f$ of any layer of $G_{1}$ induces a colouring on $G_{1}$ which cannot be broken by the automorphism $\alpha_{t}$ of $G_{1}$. Since there are infinitely many such automorphisms, we may assume without loss of generality that $\alpha_{s} \neq \alpha_{t}$ when $s \neq t$.

Now consider non-trivial automorphisms of $G_{1} \square G_{2}$ of the form $\alpha=\left(\alpha_{t}, \mathrm{id}_{G_{2}}\right)$ (for some $t \geq k$ ). For each such $t$, and each edge $v w$ of $G_{1}$ (which we can consider as an edge of any $G_{1}$-layer of $\left.G_{1} \square G_{2}\right)$, we have that $f(v w)=f\left(\alpha_{t}(v) \alpha_{t}(w)\right)$, i.e., these automorphisms of $G_{1} \square G_{2}$ are not broken by edges in layers of $G_{1}$.

The automorphisms $\alpha$ of the above form should therefore be broken by edges of layers of $G_{2}$. But this means that, for each $t \geq k$, for at least one edge $x y$ of the $G_{2}$-layer determined by a vertex $v \in V\left(G_{1}\right)$, we have that $f(x y)$ in this layer is different from $f\left(\alpha_{t}(x) \alpha_{t}(y)\right)$ in the $G_{2}$-layer determined by $\alpha_{t}(v) \in V\left(G_{1}\right)$. Since there are infinitely many $G_{2}$-layers, this requires infinitely many different colourings of $G_{2}$. However, there are at most $k^{\left\|G_{2}\right\|}$ different colourings of $G_{2}$-layers. Hence the colouring $f$ cannot break all the infinitely many automorphisms described above.

As a consequence of the above three lemmas we immediately obtain the following characterisation. 
Theorem 2.4. If $G_{1}$ and $G_{2}$ are connected relatively prime countable graphs, then $D^{\prime}\left(G_{1} \square G_{2}\right)$ is infinite if and only if for some $i \in\{1,2\}$ we have that $D^{\prime}\left(G_{i}\right)$ is infinite while for $j \neq i$ we have that $G_{j}$ is finite.

Now we consider a graph which is the Cartesian power $G^{k}$ of a denumerable graph $G$. For a finite graph $G$, the distinguishing number of the Cartesian power of $G$ is considered in [4]. Here we prove a result for graphs $G$ with a finite number of prime factors (counted with their multiplicities). We begin with a result for prime graphs.

Lemma 2.5. Let $k \geq 2$ be an integer. If a connected denumerable graph $G$ is prime with respect to the Cartesian product, then $D^{\prime}\left(G^{k}\right)=2$.

Proof. If $k=2$, the proof is similar to the proof of Lemma 2.1. Indeed, denote $G^{2}=$ $G_{1} \square G_{2}$, where $G_{1}, G_{2}$ are isomorphic to $G$. Using an analogous proof technique but colouring distinct even numbers of edges of each $G_{1}$-layer with red and distinct odd numbers of edges of each $G_{2}$-layer with red will also take care of the additional automorphisms generated by the isomorphism between $G_{1}$ and $G_{2}$.

Now we show that $D^{\prime}(G \square H)=2$ if $D^{\prime}(H)=2$ and $G$ is prime. In particular, if we consider $H=G^{k-1}$ then we obtain the thesis by induction. Namely, let $f$ be a distinguishing colouring of $H$ with two colours. We define a colouring of $G \square H$ as follows: One $H$-layer is given the colouring $f$, hence all automorphisms of this $H$-layer are broken. We colour another $H$-layer completely blue and all remaining $H$-layers we colour with distinct numbers of red edges different from the number of red edges in $f$. Hence all automorphisms of $G$ are broken. If $G^{\prime}$ isomorphic with $G$ is a factor of $H$, then we have additional automorphisms, generated by interchanging of $G$ and $G^{\prime}$. To break them, we colour each $G$-layer red. Then every $G^{\prime}$-layer contained in a blue $H$-layer is completely blue, so it cannot be interchanged with $G$. In this way we break all nontrivial automorphisms of $G \square H$ with two colours if $D^{\prime}(H)=2$ and $G$ is prime.

The above proof is analogous to the proof of a similar result in [7]. Observe that $D^{\prime}(G \square H)=2$ if $D^{\prime}(H)=2$ and $G$ is prime, also if $G$ is finite.

Theorem 2.6. Let $k \geq 2$ be an integer and $G$ be a connected denumerable graph with the prime factor decomposition $G=G_{1} \square \ldots \square G_{r}$, where $G_{1}, \ldots, G_{r}$ are not necessarily distinct. Then $D^{\prime}\left(G^{k}\right)=2$.

Proof. If $G$ is prime, the claim follows from Lemma 2.5. If $G$ is not prime, we consider the prime factorization $G=G_{1} \square \ldots \square G_{r}$ and apply Lemma 2.5 to every infinite factor ( $G$ has at least one infinite prime factor). Moreover, we can use Theorem 1.4 for every finite factor. The result then follows from Lemma 2.2 unless $G=K_{2} \square H$ and $k=2$, where $H$ is an infinite graph relatively prime with $K_{2}$. But we already know that $D^{\prime}\left(H^{2}\right)=2$ due to the above arguments, so let $f$ be a distinguishing colouring of $H^{2}$ with two colours. We then define a colouring of $G^{2}$ in terms of its four $H^{2}$-layers as follows: One $H^{2}$-layer is given the colouring $f$, hence all automorphisms of this $H^{2}$-layer are broken. The three remaining $\mathrm{H}^{2}$-layers are coloured with distinct numbers of red edges (while all remaining edges are blue), hence all automorphisms of $G^{2}$ are broken.

We say the $G$ has infinite diameter if there are vertices of arbitrarily large distance. Such a situation occurs in particular in any weak Cartesian product $G$ of infinitely many non-trivial factors (finite or infinite). Hence the above theorem immediately implies the following. 
Corollary 2.7. Let $k \geq 2$ be an integer and let $G$ be a connected denumerable graph with finite diameter. Then $D^{\prime}\left(G^{k}\right)=2$.

\section{The distinguishing index of the infinite hypercube}

The situation is quite different when we have infinitely many factors in the Cartesian power - consider for example the infinite dimensional hypercube $K_{2}^{\aleph_{0}}$. This (uncountable) graph has vertices represented by (denumerable) sequences of 0's and 1's and two vertices are adjacent whenever their binary sequences differ in exactly one entry. This graph also has uncountably many connected components, each a countable graph, which are pairwise isomorphic. The automorphism group of $K_{2}^{\aleph_{0}}$ is well described (see [10]). Using this information, we are now ready to prove

Theorem 3.1. Let $K_{2}^{\aleph_{0}}$ be the infinite dimensional hypercube. Then $D^{\prime}\left(K_{2}^{\aleph_{0}}\right)=2$.

Proof. We first construct an asymmetric spanning tree and then show how it can be used to prove the existence of an asymmetric spanning subgraph in every component of $K_{2}^{\aleph_{0}}$; these subgraphs will be constructed in such a way that different components have non-isomorphic subgraphs. Towards the end of the proof, we shall show how they can be exploited to break all non-trivial automorphisms of the hypercube $K_{2}^{\aleph_{0}}$.

It is convenient to describe the required asymmetric subgraphs by first handling the connected component $C^{0}$ in which all sequences have only finitely many 1's (and therefore an infinite tail of 0's). First we build an asymmetric tree $T$, which is a spanning subgraph of $C^{0}$, as follows:

Take $(0,0,0,0, \ldots)$ and let it be the central vertex. Then add $(1,0,0,0, \ldots)$, and the edge between it and the central vertex, to form the first branch of the tree. Next take $(0,1,0,0,0, \ldots)$ and $(1,1,0,0,0, \ldots)$ and the path between them and the central vertex to form the second branch of the tree. The $i$ 'th branch of this tree will therefore be the path on the central vertex and $\left(0^{i-1}, 1,0,0,0, \ldots\right),\left(0^{i-2}, 1,1,0,0,0, \ldots\right), \ldots$ and will have length $2^{i-1}$. All these binary sequences have 1 on the $i$ 'th entry and if we restricted them to the first $i-1$ entries, then we obtain the binary-reflected Gray code list with $i-1$ bits. It can be generated recursively from the list for $i-2$ bits by reflecting the list (i.e. listing the entries in reverse order), concatenating the original list with the reversed list, prefixing the entries in the original list with 0 , and then prefixing the entries in the reflected list with 1. In particular, the last vertex of the $i$ 'th branch has the code $\left(1,0^{i-2}, 1,0,0,0, \ldots\right)$, and the last but one has the code $\left(1,0^{i-3}, 1,1,0,0,0, \ldots\right)$.

Note that all branches of $T$ are of different length, which ensures us that $T$ is asymmetric, and note that it is a spanning tree of the component $C^{0}$. So it means that we can easily distinguish the weak Cartesian product of $\aleph_{0}$ copies of $K_{2}$ by two colors: Namely we colour all the edges of $T$ with one colour and the remaining edges with the second colour.

Now we would like to distinguish the Cartesian product of $\aleph_{0}$ copies of $K_{2}$ by two colours. Consider any sequence $\mathbf{x}=\left(x_{1}, x_{2}, \ldots\right)$ of 0 's and 1's and suppose it is in the connected component $C$ of the hypercube $K_{2}^{\aleph_{0}}$. Since $C$ is isomorphic to $C^{0}$, we can find a copy of $T$, say $T^{C}$, in $C$. Now we use $\mathbf{x}$ and $T^{C}$ to create a spanning subgraph $T_{\mathbf{x}}^{C}$ of $C$ by adding edges to $T^{C}$ as follows:

For every positive integer $i$ we add the edge of $K_{2}^{\aleph_{0}}$ between the endvertex of the $i$ 'th branch and the last but one vertex of the $(i+1)$ 'th branch of $T^{C}$ to this tree if and only if 
$x_{i}=1$. We remark that this edge is indeed in $K_{2}^{\aleph_{0}}$ since the binary sequences representing these vertices in $C^{0}$ differ in exactly one entry, namely the $(i+1)^{\prime}$ 'th entry, and therefore the same is true in the isomorphic copy $T^{C}$ of $T$. Note also that the choice of the added edges ensures us that $T_{\mathbf{x}}$ is not isomorphic to $T_{\mathbf{x}^{\prime}}$ whenever $\mathbf{x} \neq \mathbf{x}^{\prime}$. Since there are uncountably many sequences $\mathbf{x}$, we thus have uncountably many pairwise non-isomorphic subgraphs all of which are asymmetric.

Finally we prove, using these subgraphs of the components of $K_{2}^{\aleph_{0}}$, that the infinite hypercube is 2-distinguishable. Consider the following colouring $f$ of the edges of $K_{2}^{\aleph_{0}}$ : Colour, for each component $C$ of $K_{2}^{\aleph_{0}}$ and some fixed choice of a vertex x of $C$, all the edges of the spanning subgraph $T_{\mathbf{x}}^{C}$ with 1 ; colour all the other edges of $K_{2}^{\aleph_{0}}$ with 2 . Then consider any automorphism $\alpha$ of $K_{2}^{\aleph_{0}}$. Since isomorphisms, and thus $\alpha$, preserve connectivity, $\alpha$ has to take every component $C$ of $K_{2}^{\aleph_{0}}$ to a component $C^{\prime}$ of $K_{2}^{\aleph_{0}}$. But, if $C \neq C^{\prime}$, then the asymmetric spanning subgraphs $T_{\mathbf{x}}^{C}$ and $T_{\mathbf{x}^{\prime}}^{C^{\prime}}$ of $C$ and $C^{\prime}$ are not isomorphic (because $\mathbf{x} \neq \mathbf{x}^{\prime}$ ), hence the colouring $f$ breaks $\alpha$.

\section{References}

[1] M. O. Albertson and K. L. Collins, Symmetry breaking in graphs, Electron. J. Combin. 3 (1996), R18.

[2] I. Broere and M. Pilśniak, The distinguishing index of some infinite graphs, Electron. J. Combin. 23(1) (2015), P1.78.

[3] E. Estaji, W. Imrich, R. Kalinowski, M. Pilśniak and T. Tucker, Distinguishing number for the Cartesian product of countable graphs, Discuss. Mathem. Graph Th., http:// dx . doi . org/10.7151/dmgt.1902.

[4] A. Gorzkowska, R. Kalinowski and M. Pilśniak, Distinguishing the Cartesian product of finite graphs, Ars Math. Contemp. 12 (2017), 77-87.

[5] W. Imrich, R. Kalinowski, F. Lehner and M. Pilśniak, Endomorphism breaking in graphs, Electron. J. Combin. 21(1) (2014), P1.16.

[6] W. Imrich and S. Klavžar, Product Graphs, John Wiley \& Sons, Inc. New York, 2000.

[7] W. Imrich and S. Klavžar, Distinguishing Cartesian powers of graphs, J. Graph Theory $\mathbf{5 3}$ (2006), 250-260.

[8] W. Imrich, S. Klavžar and V. Trofimov, Distinguishing infinite graphs, Electron. J. Combin. 14 (2007), R36.

[9] R. Kalinowski and M. Pilśniak, Distinguishing graphs by edge-colourings, Europ. J. Combin. 45 (2015).

[10] M. Pankov, A note on automorphisms of the infinite-dimensional hypercube graph, The Electron. J. Comb. 19(4) (2012), P23.

[11] M. Pilśniak, Edge Motion and the Distinguishing Index, Preprint MD 076, http://www . ii.uj.edu.pl/premD.

[12] S. M. Smith, T. Tucker and M. E. Watkins, Distinguishability of infinite groups and graphs, Electron. J. Combin. 19 (2012), P27.

[13] T. Tucker, Distinguishing maps, Electron. J. Combin. 18 (2011), R50.

[14] M. E. Watkins and X. Zhou, Distinguishability of locally finite trees, Electron. J. Combin. 14 (2007), R29. 